\title{
Convexity of hesitant fuzzy sets
}

\author{
Vladimír Janiš ${ }^{\mathrm{a}, *}$, Susana Montes ${ }^{\mathrm{b}}$ and Magdaléna Renčovác \\ ${ }^{a}$ Department of Mathematics, Faculty of Natural Sciences, Matej Bel University, Slovakia \\ E-mail:vladimir.janis@umb.sk \\ ${ }^{\mathrm{b}}$ Department of Statistics and Operational Research, University of Oviedo, Spain \\ E-mail:montes@uniovi.es \\ ${ }^{\mathrm{c}}$ Department of Mathematics, Faculty of Natural Sciences, Matej Bel University, Slovakia \\ E-mail: magdalena.rencova@umb.sk
}

\begin{abstract}
We show that a definition of convexity based on the convexity of the score function does not guarantee preservation of convexity under intersections and provide a concept of convexity for hesitant fuzzy sets without this backdraw. We study the relationship between convex hesitant fuzzy sets and convex rough sets as their cuts.
\end{abstract}

Keywords: Fuzzy sets, hesitant fuzzy sets, convexity, rough sets.

\section{Introduction}

Hesitant fuzzy sets are one of currently studied generalizations of fuzzy sets (and in fact a special case of type-2 fuzzy sets). A natural motivation for such objects is a mathematical model for an evaluation by a group of (perhaps mutually dependent) experts. The potential dependence justifies a hesitant fuzzy set as a single object, similar to IF-sets, that could also be studied as pairs of fuzzy sets, but due to their nature and motivation, this is not the proper case how to understand an IF-set. Formally hesitant fuzzy sets have been introduced in [6] and [7], but the idea itself can be found also in previous works, like [2]. Although they are a relatively new object of research, they seem to have high potential for applications, especially in decision making (see $[9,10,11,12])$.

Convexity, as one of the crucial geometrical concepts, is important also for fuzzy sets theory and its applications, like fuzzy optimization. An attempt to define convexity for hesitant fuzzy sets has been done in [4], however, as we will show, there are some problems connected to the concept of convexity used there. In this work we show an alternative definition that re-

${ }^{*}$ Corresponding author. Tel.:+421 948998 759; E-mail: vladimir.janis@umb.sk. spect some expected properties of convex sets. As a basis for our consideration we take the usual definition of convexity for fuzzy sets (see [1]), i.e. a fuzzy set $f$ defined on a linear space $X$ is convex, if for each $x, y \in X, \lambda \in[0,1]$ there is

$$
f(\lambda x+(1-\lambda) y) \geq \lambda f(x)+(1-\lambda) f(y) .
$$

Clearly the convexity of a fuzzy set is equivalent to convexity of all its $\alpha$-cuts $f_{\alpha}=\{x \in x ; f(x) \geq \alpha\}$.

\section{Basic concepts}

A hesitant fuzzy sets (HFS) is defined in terms of functions that returns a set of membership values for each element in the domain. Throughout this work we will use HFS in two different meanings, as an abbreviation of the words "hesitant fuzzy set" and also as the set of all hesitant fuzzy sets defined on the real line. Thus, the expressions " $f$ is a HFS" and " $f \in H F S "$ mean the same. We can define a HFS in two different points of view. First, a HFS can be defined as an extension of fuzzy sets, as introduced in [6].

Definition 1 Let $X$ be a reference set, a HFS on $X$ is a function $h$ that assigns a subset of $[0,1]$ to $x \in X$, i.e. $h: X \rightarrow 2^{[0,1]}$. 
Hence HSF provide a general frame, covering e.g. fuzzy sets, interval-valued sets and formally also IFsets.

Sometimes this definition is restricted in some way, mostly it is assumed that the values $h(x)$ are finite subsets of the unit interval.

Another possible definition of HFS is based on its concept of (possibly mutually interrelated) set of fuzzy sets.

Definition 2 Let $X$ be a reference set and $A$ be a set of membership functions. The HFS associated with A, $h^{A}$, is defined as $h^{A}: X \rightarrow 2^{[0,1]}$ such that

$$
h^{A}(x)=\bigcup_{\mu \in A}\{\mu(x)\}
$$

Again, the set $A$ can be supposed to be finite. This definition is quite suitable in decision making, when experts assess a set of alternatives. In such case, each element of $A$ represents the assessments of an individual expert of all alternatives and $h^{A}(x)$ represents the set of assessments of the the experts for the single alternative $x$.

To score function for a hesitant fuzzy set $h$ with a finite set of values at each $x$ has been introduced in [8] in the following way:

Definition 3 [8] For $h \in H F S$ the function $s_{h}: X \rightarrow$ $[0,1]$

$$
s_{h}(x)=\frac{1}{l_{h}(x)} \sum_{\gamma \in h(x)} \gamma
$$

where $l_{h}(x)$ is the number of the elements in $h(x)$, is called a score function of $h$.

The score function is also used for the definition of cuts of a HFS, as by an $\alpha$-cut of a HFS we can understand the respective $\alpha$-cut of its score function.

\section{Convexity based on a score function}

Rashid and Beg in [4] introduced the following the concept of convexity for HFS.

Definition 4 [4] Let $X$ be a vector space. Then $h \in$ $H F S$ with a score function $s_{h}$ is said to be quasi-convex if for all $x, y \in X, \lambda \in[0,1]$ it holds that $s_{h}(\lambda x+(1-$ $\lambda) y)) \geq \min \left\{s_{h}(x), s_{h}(y)\right\}$.
In [4] it is also shown that the system of all quasiconvex HFS is exactly the system of all HFS with convex cuts (e.g. corresponding cuts of the score function), which means that the definition is at least from this point of view reasonable. Moreover, if $f$ is a convex fuzzy set, then it is also convex with respect to [4] as a HFS with singleton values.

However, one of the principal properties, that makes the collection of all convex sets so important in a variety of applications, like e.g. optimization (see $[1,5]$ ) is the fact, that convexity is preserved under arbitrary intersections. As we will show in a counter-example, the concept of quasi-convexity defined in [4] has not this property.

Let us assume that we restrict ourselves to HFS with finite values. To work with intersections, we will repeat the notation form [6]: Suppose $f, g \in H F S$. Then $f^{+}(x)=\max \{f(x)\}, f^{-}(x)=\min \{f(x)\}$. The intersection of $f, g$ is the following HFS:

$(f \cap g)(x)=\left\{\gamma \in\{f(x) \cup g(x)\} ; \gamma \leq \min \left\{f^{+}(x), g^{+}(x)\right\}\right\}$

Later we will use the following identities, which are direct consequences of the above definition:

$$
(f \cap g)^{+}=\min \left\{f^{+}, g^{+}\right\}, \quad(f \cap g)^{-}=\min \left\{f^{-}, g^{-}\right\}
$$

In the following example we show that the intersection of two quasi-convex HFS (using the definition of quasi-convexity from [4]) need not be a quasi-convex HFS.

Example 1 Let $f, g$ be HFS, both defined on the interval $[-1,1]$, each consisting of two membership functions: $f_{1}(x)=0.1, f_{2}(x)=0.9$ (full lines) and $g_{1}(x)=$ $1-\frac{|x|}{5}, g_{2}(x)=0.9-\frac{|x|}{5}$ (dashed lines).

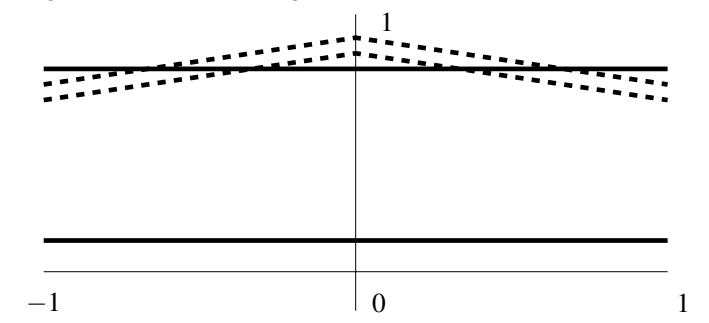

Their score functions are $s_{f}(x)=0.5, s_{g}(x)=0.95-$ $\frac{|x|}{5}$. Hence, both $f, g$ are quasi-convex HFS. Their intersection is the following: 


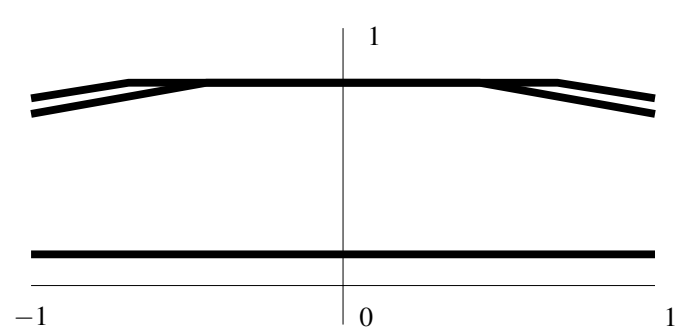

The value of its score function at 0 is $\frac{1}{2}(0.1+0.9)=$ 0.5 , but the value of the score function at both points -1 and 1 is $\frac{1}{3}(0.1+0.7+0.8)=0.533 \cdots>0.5$, hence this intersection is not quasi-convex.

The previous example shows that it is necessary to provide an alternative definition of convexity for HFS - a convexity, which is at least preserved under intersections.

Another weak point of the attitude in [4] is that it cannot be used for HFS with infinite sets as values, unless the score function is redefined.

\section{Convexity preserved under intersections}

Our aim is to provide a definition of convexity for hesitant fuzzy sets that fulfill the following natural conditions:

- a convex fuzzy set considered as a HFS with singleton values is convex,

- an intersection of convex HFS is a convex HFS,

- a (reasonable defined) cut of a convex HFS is a convex set.

To find the proper definition we may be guided by the principle of convexity for fuzzy sets on a real interval, which says that a fuzzy set is convex on an interval $[x, z]$ if and only if for each $y$ such that $x<y<z$ there is $f(y) \geq \min \{f(x), f(z)\}$. Thus we can consider the following options for convexity of a hesitant fuzzy set $f$, again for points $x<y<z$ :

- all the values of $f(y)$ are greater or equal to all the values of either $f(x)$ or $f(z)$,

- at least one value of $f(y)$ is greater or equal to all the values of either $f(x)$ or $f(z)$,

- at least one value of $f(y)$ is greater or equal to at least one value of either $f(x)$ or $f(z)$.

Although formally we can consider convexity in any of the above cases, the first one is too restrictive, as under this condition a HFS consisting of two different constant membership functions would not be convex.
Also the last option is not too practical, because then any HFS containing a constant membership function would be convex. Thus we will concentrate on HFS fulfilling the second option and of course all three conditions for convexity stated at the beginning of this section.

As we will show, the following definition fulfills all these requirements.

Definition 5 Let $f \in H F S$ in a vector space $X$. Then $f$ is convex if for all $x, z \in X, \lambda \in[0,1]$ there is

$$
f^{+}(y) \geq \min \left\{f^{+}(x), f^{+}(z)\right\}
$$

where $y=\lambda x+(1-\lambda) z$.

It is immediate, that a convex fuzzy set is also a convex HFS, as in such case $f=f^{+}$.

In the following proposition we show that convexity is preserved under intersections.

Proposition 1 Let $f, g \in H F S$ in a vector space $X, f, g$ convex. Then $f \cap g$ is also convex.

Proof. Suppose $f, g$ are convex HFS on $X$ and $x, z \in$ $X, y=\lambda x+(1-\lambda) z, \lambda \in[0,1]$. We will show the inequality

$$
(f \cap g)^{+}(y) \geq \min \left\{(f \cap g)^{+}(x),(f \cap g)^{+}(z)\right\} .
$$

As we have noted at the definition of the intersection for HFS, we have

$$
\begin{aligned}
& (f \cap g)^{+}(y)=\min \left\{f^{+}(y), g^{+}(y)\right\} \geq \\
& \geq \min \left\{\min \left\{f^{+}(x), f^{+}(z)\right\}, \min \left\{g^{+}(x), g^{+}(z)\right\}\right\}= \\
& =\min \left\{\min \left\{f^{+}(x), g^{+}(x)\right\}, \min \left\{f^{+}(z), g^{+}(z)\right\}\right\}= \\
& =\min \left\{(f \cap g)^{+}(x),(f \cap g)^{+}(z) .\right.
\end{aligned}
$$

Hence the intersection of two convex HFS is convex.

We can also see, that at least one value of $f(y)$ (for example $\left.f^{+}(y)\right)$ is greater or equal to all the values of either $f(x)$ or $f(z)$.

Finally it will also be interesting to establish the connection between convex HFS and their cuts. If for $\alpha \in[0,1]$ we define an $\alpha$-cut of $f \in H F S$ as the set

$$
f_{\alpha}=\{x \in X ; \text { there exists } \gamma \in f(x) ; \gamma \geq \alpha\}
$$

then we have $f_{\alpha}=f_{\alpha}^{+}$and the equivalence between the convexity of $f$ and convexity of its cuts is immediate.

If we strengthen the condition of convexity in Definition 5 adding the condition

$$
f^{+}(y) \geq \min \left\{f^{-}(x), f^{-}(z)\right\}
$$


(note that the analogical statement to Proposition 1 remains valid), we obtain a proper subset of all convex HFS, which we can call strongly convex HFS.

Also we can define a strong $\alpha$-cut of a HFS $f_{\alpha}^{*}$ as the set

$$
f_{\alpha}^{*}=\{x \in X ; \text { for all } \gamma \in f(x) ; \gamma \geq \alpha\}
$$

Now we have a connection of strongly convex HFS and rough sets, that were defined in [3]. We remind that by a rough set in a universe $X$ we understand a pair $(A, B) \in 2^{X} \times 2^{X}$ such that $A \subseteq B$. A rough set is convex if both its parts are convex.

If for some $\alpha$ all the values $\gamma \in f(x)$ exceed $\alpha$, then also $f^{-}(x) \geq \alpha$ and vice versa. Thus $f_{\alpha}^{*}=f_{\alpha}^{-}$and we have the following proposition:

Proposition 2 Let $f$ be a HFS. Then $f$ is strongly convex if and only of all its pairs of cuts $\left(f_{\alpha}^{*}, f_{\alpha}\right)$ are convex rough sets.

\section{Concluding remarks}

We have provided a definition of convexity for hesitant fuzzy sets that preserves convexity under intersection. Of course it would be better to show immediately, that the intersection of an arbitrary collection of convex HFS is a convex HFS. In Proposition 1 we preferred to work with just two HFS, partly or clearness, partly because the intersection in [6] is also defined for two HFS. However, the generalization is obvious. First, for any $f \in H F S$ (no condition on values) we can define $f^{+}(x)=\sup f(x)$ and $f^{-}(x)=\inf f(x)$. Then if $\Phi$ is an arbitrary set of indices, we put

$$
\cap_{\varphi \in \Phi}\left(f_{\varphi}\right)(x)=\left\{\gamma \in \cup_{\varphi \in \Phi} f_{\varphi}(x) ; \gamma \leq \inf f_{\varphi}^{+}(x)\right\} \text {. }
$$

The same method as in the proof of Proposition 1 shows that the intersection of arbitrary convex HFS is a convex HFS.

In Definition 5 we could also use the inequality $f^{-}(y) \geq \min \left\{f^{-}(x), f^{-}(z)\right\}$, i.e. to require the convexity of the "lower envelope". The convexity of the intersection in such case is even more obvious, as it follows from the convexity of the minimum of two convex fuzzy sets.
Comparing with the method used in [4] we admit that the advantage of a score function is that it aggregates all the values of $f(x)$, while convexity in our attitude depends only on the convexity of $f^{+}$, or (in case of the strong convexity) of $f^{+}$and $f^{-}$. In future research it will be interesting to discuss systems of convex HFS, where the convexity is connected also with the intermediate values of $f(x)$.

\section{Acknowledgement}

The research in this communication has been supported in part by MINECO-TIN2014-59543-P its financial support is gratefully acknowledged.

\section{References}

[1] Ammar E., Metz J.: On fuzzy convexity and parametric fuzzy optimization, Fuzzy Sets and Systems 49 (1992), pp. 135-141.

[2] Grattan-Guinness, I.: Fuzzy membership mapped onto interval and many-valued quantities, Zeitschrift fur mathematische Logik und Grundladen der Mathematik 22 (1976) 149-160.

[3] Pawlak, Z.: Rough sets, Int. J. Inf. Comp. Sci. 11:341356(1982)

[4] Rashid T., Beg, I.: Convex hesitant fuzzy sets, Journal of Intelligent and Fuzzy Systems 30 (5) (2016), pp. 2791-2796.

[5] Syau, Y., Lee, E.S.: Fuzzy convexity with application to fuzzy decision making, in: Proceedings of the 42nd IEEE Conference on Decision and, Control, 2003, pp. 5221-5226.

[6] Torra, V.: Hesitant fuzzy sets, International Journal of Inteligent Systems 25 (6) (2010), pp. 529-539.

[7] Torra, V., Narukawa, Y.: On hesitant fuzzy sets and decision, in: Proceedings of the 18th IEEE International Conference on Fuzzy Systems, Jeju Island, Korea (2009) 1378-1382.

[8] Xia, M. and Xu, Z.: Hesitant fuzzy information aggregation in decision making, Int. Journ. of Approximate Reasoning 52 (2011), pp. 395-407.

[9] Xu, Z.S., Xia, M., Chen, N.: Some Hesitant Fuzzy Aggregation Operators with Their Application in Group Decision Making, Group Decision and Negotiation, 22(2)(2013) 259-279.

[10] Wei, G.W, Zhang, N.: A multiple criteria hesitant fuzzy decision making with Shapley value-based VIKOR method, Journal of Intelligent and Fuzzy Systems, 26(2) (2014) 1065-1075.

[11] Wei, G.W., Wang, H.J., Zhao, X.F., Lin, R.: Approaches to hesitant fuzzy multiple attribute decision making with incomplete weight information, Journal of Intelligent and Fuzzy Systems, 26(1) (2014) 259-266.

[12] Zhang, Z.: Hesitant fuzzy power aggregation operators and their application to multiple attribute group decision making. Inf. Sci. 234: 150-181 (2013) 